\title{
DOENÇA DO REFLUXO GASTROESOFÁGICO: QUAL A EFICÁCIA DOS EXAMES NO DIAGNÓSTICO?
}

\author{
Gastroesophageal reflux disease: what is the effectiveness of diagnostic tests?
}

\author{
Nelson Adami ANDREOLLO, Luiz Roberto LOPES, João de Souza COELHO-NETO
}

Trabalho realizado na Disciplina de Moléstias do Aparelho Digestivo e Gastrocentro, Departamento de Cirurgia, Faculdade de Ciências Médicas da UNICAMP, Campinas, SP, Brasil

DESCRITORES - Refluxo gastroesofágico. Endoscopia do sistema digestório. Cintilografia.

Correspondência:

Nelson Adami Andreollo, e-mail: nandreollo@hotmail.com

Fonte de financiamento: não há Conflito de interesses: não há

Recebido para publicação: 22/07/2009 Aceito para publicação: 12/01/2010

HEADINGS - Gastroesophageal reflux. Endoscopy, digestive system. Radionuclide imaging.
RESUMO - Racional - A doença do refluxo gastroesofágico (DRGE) é muito frequente na população e os exames empregados para o seu diagnóstico são a endoscopia digestiva alta, exames radiológicos contrastados, a cintilografia com tecnécio99m Tc, a manometria e a phmetria de 24 h. Objetivo - Analisar a eficácia destes exames em doentes portadores de DRGE submetidos a tratamento cirúrgico por vídeolaparoscopia. Métodos - Estudo retrospectivo de 126 doentes com diagnóstico de DRGE, que realizaram no pré-operatório endoscopia digestiva alta, exame radiológico do esôfago, estômago e duodeno, cintilografia e manometria do esôfago. A phmetria de 24 h foi realizada em número pequeno de doentes. Resultados - A endoscopia digestiva diagnosticou esofagite de refluxo em 91,7\% dos casos e hérnia hiatal em $88,6 \%$. Os exames radiológicos demonstraram a presença de refluxo gastroesofágico em $80,2 \%$ dos casos e hérnia hiatal em $91,5 \%$. A cintilografia demonstrou refluxo gastroesofágico em $85,1 \%$ dos casos. A manometria esofágica foi normal em $6,45 \%$ dos casos. Conclusão - O exame subsidiário que apresentou elevada sensibilidade para o diagnóstico da esofagite de refluxo foi a endoscopia digestiva alta; o estudo cintilográfico foi o e que mais demonstrou refluxo gastroesofágico; o exame radiológico contrastado foi o de melhor eficácia em demonstrar o refluxo.
ABSTRACT - Background - Gastroesophageal reflux disease (GERD) is widespread in the population and the tests used for diagnosis are endoscopy, radiological contrast, scintigraphy with $99 \mathrm{~m} \mathrm{Tc}$, manometry and $24 \mathrm{~h}$ phmetry. Aim - To analyze the effectiveness of these tests in patients with GERD who underwent surgical treatment by video-laparoscopy. Methods - A retrospective study of 126 patients diagnosed with GERD, who underwent preoperative upper gastrointestinal endoscopy, radiologic examinations of the esophagus, stomach and duodenum, scintigraphy and manometry of the esophagus. The 24 hours phmetry was performed in small number of patients. Results - Endoscopy reflux esophagitis was diagnosed in $91.7 \%$ of cases and hiatus hernia in $88.6 \%$. Radiological examinations showed the presence of gastroesophageal reflux in $80.2 \%$ and hiatus hernia in $91.5 \%$. Scintigraphy showed gastroesophageal reflux in $85.1 \%$. Esophageal manometry was normal in $6.45 \%$. Conclusion Upper digestive endoscopy presented high sensitivity for the diagnosis of reflux esophagitis, but scintigraphy was better showing the reflux, so did contrasted radiographic examination.

\section{INTRODUÇÃO}

$\mathrm{N}$ as últimas três décadas, as investigações das hérnias hiatais e da doença do refluxo gastroesofágico (DRGE) têm se concentrado nas anormalidades fisiológicas responsáveis pelo refluxo, utilizando exames aperfeiçoados e eficazes e, portanto, resultados mais fidedignos e seguros. As hérnias hiatais, quando pesquisadas, são encontradas em porcentagem significativa da população adulta, podendo ser assintomáticas ${ }^{2,5}$.

O refluxo gastroesofágico sintomático também é muito comum nos dias atuais, com pirose diária relatada por $11 \%$ da população, além de 12 e $15 \%$, respectivamente, com sintomas mensais ou semanais. Embora a grande 
maioria dessas pessoas não necessite de tratamento, a DRGE com ou sem hérnia hiatal é certamente um problema comum nos consultórios e hospitais ${ }^{9,11}$.

A junção esofagogástrica é uma zona de alta pressão de 3 a $4 \mathrm{~cm}$ de comprimento, localizada logo acima da junção do esôfago com o estômago. O arranjo muscular a esse nível cria assimetria radial, com pressões mais altas medidas na parede lateral esquerda. O esfíncter esofágico inferior (EEI) é composto de músculo liso e está sob controle não-colinérgico e não adrenérgico. Ele é mantido em estado de contração constante, principalmente devido à atividade miogênica extrínseca, mas seu tônus de repouso é afetado por vários fatores neurais e hormonais ${ }^{3}$.

O tônus do esfíncter aumenta em resposta às pressões intra-abdominais aumentadas e durante as contrações gástricas. O EEI relaxa ao mesmo tempo conforme a onda peristáltica se aproxima. A peristalse esofágica empurra o bolo alimentar na luz esofágica em direção à cavidade gástrica e o bolo alimentar atravessa o esfíncter relaxado, sendo que imediatamente este se fecha tão logo atinja o estômago. As pressões de fechamento do esfíncter são o dobro dos seus valores, após o que retornam aos níveis de repouso. O seu relaxamento ocorre do mesmo modo que com a peristalse secundária. Após as refeições, quando o estômago está cheio, ocorrem relaxamentos transitórios espontâneos, sem relação com a peristalse, durando de cinco a 30 segundos. É durante esses relaxamentos transitórios que grande parte do refluxo ocorre, tanto em indivíduos normais como em pacientes com DRGE ${ }^{3,4}$.

Outros fatores além do tônus do EEI extrínseco desempenham papel na prevenção do refluxo gastroesofágico. A capacidade do esôfago de esvaziar seu conteúdo (clearance) varia entre os indivíduos normais. Recebe influência da posição, consistência do bolo alimentar e pH dentro do esôfago. O epitélio escamoso normalmente é impermeável ao íon hidrogênio. A exposição a ácidos, especialmente quando misturados a sais biliares e enzimas pancreáticas, causa perda da resistência da mucosa, podendo resultar em difusão retrógrada do ácido através da mucosa ${ }^{4,12}$.

A organização muscular do esfíncter, o comprimento do esôfago intra-abdominal, o ângulo agudo de entrada da junção esofagogástrica e sua função valvar (ângulo esofagogástrico), bem como a integridade das estruturas de suporte para manter a posição correta dentro do hiato, desempenham papel importante na prevenção do refluxo gastroesofágico. Mittal $(1993)^{12}$ observou que o pilar diafragmático apresenta capacidade especial de compressão que ajuda a aumentar as pressões na junção esofagogástrica, principalmente durante a inspiração e quando ocorre aumento de pressão intra-abdominal. A perda dessa capacidade, quando o hiato está alargado como ocorre em uma hérnia hiatal, pode predispor a refluxo, principalmente se houver perda do tônus do esfíncter.
Indivíduos normais podem apresentar refluxo fisiológico diário, sem que isso cause sintomas ou alterações fisiológicas. Quando o refluxo causa sintomas, seja pela quantidade, alteração na composição ou aumento na frequência, diz-se então que a entidade patológica da doença do refluxo gastroesofágica (DRGE) está presente. Os movimentos peristálticos primários e secundários do esôfago também são mecanismos de defesa bastante eficazes contra o refluxo. A motilidade anormal que afeta a função esofágica é observada em pacientes com hérnias hiatais sintomáticas, ao passo que apenas minoria dos pacientes com DRGE apresenta defeito de peristalse. Existe, portanto, deficiência e perda da força de peristalse com o aumento do dano à mucosa. Quando o esôfago apresenta extensa área circunferencial de epitélio colunar, observa-se aumento na peristalse deficiente e na incidência de contrações terciárias em resposta à deglutição. Tal perda de função sugere ocorrência de exposição mais prolongada a qualquer agente causador de refluxo, com prejuízo do clearance e da capacidade de esvaziamento do esôfago ${ }^{3,10}$.

Pesquisas experimentais demonstram que a infusão de ácido clorídrico causa lesão na mucosa esofágica, sendo mais agressiva quando misturado a pepsina, bile e sais biliares. As enzimas digestivas induzem a perda de sensibilidade do epitélio escamoso. A mistura de ácido e bile está presente nas formas mais graves da doença do refluxo ${ }^{3}$.

A esofagite endoscópica ocorre na maioria dos doentes com manifestações características de DRGE, ao passo que o refluxo é bem menos frequente nos doentes com manifestações atípicas. Os exames subsidiários indicados, mediante anamnese cuidadosa de caso à caso são: endoscopia digestiva alta, radiografias contrastadas do esôfago/estômago e duodeno, manometria esofágica, cintilografia e phmetria de 24 horas $^{5}$.

O objetivo deste trabalho é analisar a eficácia da endoscopia digestiva alta, exames radiológicos contrastados, cintilografia com tecnécio ${ }^{99} \mathrm{Tc}$, manometria e phmetria de $24 \mathrm{~h}$ nos portadores de DRGE submetidos a tratamento cirúrgico por vídeolaparoscopia.

\section{MÉTODOS}

Os pacientes em estudo foram encaminhados à Gastrocirurgia-HC-Unicamp devido à falha terapêutica com tratamento clínico para DRGE. O estudo foi retrospectivo e as informações obtidas dos prontuários de 132 doentes submetidos a cirurgia vídeo-laparoscópica de 1993 a 1999 para tratamento da DRGE. As informações obtidas foram: idade, sexo, tempo de evolução do início dos sintomas até a operação, resultado de laudos dos exames subsidiários presentes nos prontuários. 
Foram incluídos nesta pesquisa doentes estudados e encaminhados para tratamento cirúrgico (vídeo-laparoscopia) devido à falha terapêutica com o tratamento clínico, possuíam quadro clínico compatível com DRGE, sendo que tinham dois ou mais exames subsidiários que confirmavam a doença.

Foram excluídos os pacientes pediátricos $(0-18$ anos), os com doenças sistêmicas de base (neurológicas, reumáticas) e aqueles que possuíam menos de dois exames subsidiários que confirmassem a doença.

Os dados obtidos foram colocados no banco de dados Epi-Info 6.0 para que pudesse ser feita a análise estatística $(P<0,005-5 \%)$.

\section{RESULTADOS}

\section{Análise descritiva geral}

Em relação ao sexo, os 132 pacientes incluídos na pesquisa foram distribuídos em 65 (49,2\%) mulheres e 67 (50,8\%) homens, sem diferença estatística entre eles.

A faixa etária variou entre 19 e 78 anos, sendo a média $51,42 \pm 13,84$ anos.

Ao analisar-se os prontuários, foi constatado haver referências às queixas de refluxo em 126 casos (95,45\%), variando entre 3 e 360 meses, com tempo médio de história da doença de 86,13 $\pm 72,05$ meses ou 7,16 \pm 6 anos. Foi considerado como duração dos sintomas o período entre o início do quadro clínico até a data da operação. $\mathrm{Na}$ avaliação clínica, o tempo de apresentação dos sintomas foi considerado mais importante.

\section{Achados endoscópicos}

A endoscopia não informa em definitivo que o doente possui ou não a DRGE, mas permite visualizar alterações na mucosa esofágica causadas pela doença. Foi o exame subsidiário realizado com maior frequência pelos doentes; $100 \%$ fizeram-no, apresentando variados graus de esofagite ou hérnia de hiato.

A Tabela 1 mostra a frequência e porcentagem dos graus de esofagite segundo Savary-Müller. A Tabela 2 mostra a frequência e porcentagem de hérnia de hiato.

TABELA 1- Frequência e porcentagem dos diversos graus de esofagite de refluxo pela endoscopia (Classificação de Savary-Müller)

\begin{tabular}{|c|c|c|}
\hline GRAU DA ESOFAGITE & FREQUÊNCIA & PORCENTAGEM \\
\hline Sem esofagite & 11 & $8,3 \%$ \\
\hline 1 & 39 & $29,5 \%$ \\
2 & 25 & $18,9 \%$ \\
3 & 16 & $12,1 \%$ \\
\hline 4 & 41 & $31,1 \%$ \\
\hline
\end{tabular}

TABELA 2 - Frequência e porcentagem da hérnia de hiato pela endoscopia

\begin{tabular}{ccc}
\hline HÉRNIA DE HIATO & FREQUÊNCIA & PORCENTAGEM \\
\hline Presente & 117 & $88,6 \%$ \\
Ausente & 15 & $11,4 \%$ \\
\hline
\end{tabular}

\section{Achados radiológicos}

Foram encontrados 119 pacientes (90,2\%) que realizaram exame radiológico contrastado do esôfago, estômago e duodeno, sendo que destes, 111 (93,3\%) apresentaram alguma alteração radiológica como hérnia de hiato ou esofagite de refluxo. Há controvérsias na literatura quanto à especificidade da graduação da esofagite de refluxo por este método. Devido a isso, não consideraram-se os graus referidos nos laudos destes exames e registrou-se apenas se havia ou não positividade do exame quanto à esofagite. As Tabelas 3 e 4 mostram a frequência e porcentagem da esofagite e hérnia de hiato verificados por este exame.

TABELA 3 - Frequência e porcentagem de esofagite de refluxo demonstradas pelo estudo radiológico

\begin{tabular}{c|cc|}
\hline ESOFAGITE & FREQUÊNCIA & PORCENTAGEM \\
\hline Presente & 93 & $80,2 \%$ \\
Ausente & 23 & $19,8 \%$ \\
\hline
\end{tabular}

\section{Achados cintilográficos}

Dentre os 132 pacientes analisados, 93 (70,5\%) realizaram exame cintilográfico empregando 0 tecnésio 99 para demonstrar refluxo. Destes, 75 (80,6\%) apresentaram refluxo gastroesofágico. A Tabela 5 mostra a frequência e porcentagem dos diferentes graus de refluxo.

TABELA 4 - Frequência e porcentagem da hérnia de hiato demonstrada pelo estudo radiológico

\begin{tabular}{ccc}
\hline HÉRNIA DE HIATO & FREQUÊNCIA & PORCENTAGEM \\
\hline Presente & 107 & $91,5 \%$ \\
Ausente & 10 & $8,5 \%$ \\
\hline
\end{tabular}

TABELA 5 - Frequência e porcentagem de refluxo gastroesofágico pela cintilografia

\begin{tabular}{ccc}
\hline GRADUAÇÃO & FREQUÊNCIA & PORCENTAGEM \\
\hline Sem refluxo & 13 & $14,8 \%$ \\
\hline Grau I & 26 & $29,5 \%$ \\
\hline Grau II & 15 & $17,0 \%$ \\
\hline Grau III & 34 & $38,6 \%$ \\
\hline
\end{tabular}

\section{Achados manométricos}

Apenas 31 pacientes realizaram manometria esofágica no pré-operatório, sendo que os resultados obtidos estão na Tabela 6.

TABELA 6 - Achados manométricos

\begin{tabular}{ccc}
\hline RESULTADOS & FREQUÊNCIA & PORCENTAGEM \\
\hline Normal & 2 & $6,45 \%$ \\
\hline $\begin{array}{c}\text { Hipotonia do esfíncter } \\
\text { esofágico inferior }\end{array}$ & 26 & $29,5 \%$ \\
\hline Outros achados & 6 & $19,35 \%$ \\
\hline
\end{tabular}

\section{Achados pHmétricos}

Este exame foi realizado em cinco (16,13\%) doentes, apenas. Todos mostraram positividade, ou seja, refluxo gastroesofágico patológico. 


\section{DISCUSSÃO}

O quadro clínico de doentes com DRGE dividese em três categorias clínicas principais. A primeira é composta por sintomas típicos mais comuns, como azia e regurgitação que pioram após as refeições quando o paciente se deita e/ou se curva. A segunda, relaciona-se com as complicações da DRGE (disfagia leve na ausência de qualquer estreitamento; odinofagia; hematêmese e melena). A terceira categoria inclui disfagia orofaríngea, asma, dor torácica recorrente e faringite. A halitose, a salivação amarga, o gosto ácido na boca e problemas dentários devem ser considerados inespecíficos e não obrigatoriamente associados com o refluxo ${ }^{8}$.

O quadro clínico em casos de hérnias maiores é causado principalmente pelo seu volume no mediastino. A plenitude pós-prandial, o desconforto ou dor retroesternal e a regurgitação podem ocorrer após a alimentação. Disfagia aguda com dor torácica ou epigástrica intensas podem sugerir risco de estrangulamento ${ }^{9}$

Quando um paciente apresenta sintomas da DRGE sem anormalidades anatômicas na junção gastroesofágica, supõe-se que alterações motoras sejam as responsáveis pelos sintomas. O exemplo característico é o portador de esclerodermia que apresenta disfagia e achados radiológicos mostrando refluxo com a cárdia aberta sem hérnia mas com sintomas de esofagite importantes.

O exame radiológico contrastado do esôfago, estômago e duodeno tem papel importante na definição das anormalidades anatômicas presentes quando a hérnia de hiato e a DRGE coexistem. Além disso, também pode mostrar as alterações da mucosa e as complicações da parede esofágica presentes com a DRGE. A simples observação de refluxo de bário para dentro do esôfago, por si só, não significa que a DRGE esteja presente. Quando se comparam indivíduos normais com doentes com esofagite endoscópica, o refluxo livre radiológico é visto em $20 \%$ dos controles e em $25 \%$ dos pacientes com esofagite documentada. A mucosa colunar em esôfago distal é suspeita quando pregas semelhantes às gástricas ascendem para dentro do tórax. Eventuais distúrbios motores e digestivos, como as estenoses esofágicas, também podem ser identificados por este exame $^{1,7,9}$. O estudo radiológico é importante por demonstrar não apenas a presença de hérnia hiatal mas também o tipo, sua extensão e também a presença do refluxo, de estenose e outras anormalidades, incluindo gastroduodenais, possivelmente relacionadas ao refluxo. Neste trabalho, foi registrado que aproximadamente $80 \%$ dos doentes tiveram o seu refluxo diagnósticado pelo estudo radiológico. Além disso, em 91,5\% dos casos o estudo radiológico convencional demonstrou a presença de hérnia hiatal concomitante.

A endoscopia digestiva alta permite tanto a realização de biópsias de áreas suspeitas como de outros procedimentos terapêuticos. Deve, no entanto, ser bem indicada, uma vez que consiste em exame de custo não desprezível, especializado e invasivo.

O estadiamento do DRGE através de endoscopia visa registrar a forma, clínica ou anatômica, em determinado momento da fase evolutiva da doença, o aqui utilizado foi o de Savary-Miller ${ }^{6,9,13}$.

O diagnóstico de esofagite pode ser sugerido pelo exame radiológico mas deve ser confirmado pela endoscopia e estudo histopatológico. A ulceração pode assumir diversas formas, dimensões e profundidades, causando ou não posteriormente, estenose da junção esofagogástrica. Sua importância consiste no fato de que a conduta terapêutica é quase sempre cirúrgica, não havendo tratamento medicamentoso exclusivo ${ }^{1,7,10}$.

Nesta pesquisa, apenas $8,3 \%$ dos doentes não apresentava esofagite ao exame endoscópico, sendo que todos os demais tinham seus vários graus. Por outro lado, o exame mostrou positividade para hérnia hiatal em $88,6 \%$ dos casos.

A manometria é o exame mais indicado para detectar as alterações do peristaltismo esofágico. Atualmente, é realizada com equipamentos computadorizados e muito sensíveis, utilizando sondas de oito canais, o que possibilita a quantificação da sua função motora e pode evidenciar, ou não, anormalidades na sua motilidade, associadas a episódios de refluxo. Pressões baixas do EEI estão associadas com episódios espontâneos de refluxo e a esofagite é mais intensa quando o tônus do EEI está muito baixo. A pressão mais baixa do esfíncter tende a ser encontrada nos pacientes com estenose ou com esôfago de epitélio colunar de Barrett. A esofagite ativa altera a função de maneira proporcional à gravidade da lesão. Pacientes com esofagite grave apresentam incidência aumentada de peristalse alterada e ineficiente ${ }^{1,7,14}$. Nesta pesquisa, em $6,45 \%$ dos doentes que realizaram o exame tiveram o esfincter inferior do esôfago considerado normal.

A monitorização do $\mathrm{pH}$ nas 24 horas é o melhor meio para comprovar a presença de ácido na luz esofágica. Entretanto, este exame não detecta com exatidão o refluxo alcalino, que pode atingir porcentagem elevada de doentes ${ }^{6,8,9}$. É indicada para confirmar ou afastar refluxo em doentes com quadro clínico compatível com DRGE, porém sem esofagite, para caracterizar o padrão de refluxo, na recidiva de sintomas no pós-operatório, no esôfago de Barrett e na avaliação do tratamento clínico. Entretanto, não consegue constatar a presença de refluxo "não-ácido", sendo portanto positivo em cerca de $75 \%$ dos doentes com DRGE ${ }^{8,9}$. Nesta pesquisa os doentes que realizaram phmetria de $24 \mathrm{~h}$ foi muito pequeno, não sendo sufriciente para conclusões.

Pode-se detectar e quantificar o refluxo gastroesofágico através de técnicas cintilográficas, com a grande vantagem de ser método não invasivo e bastante sensível, fornecendo resultados semi- 
quantitativos. O fármaco mais utilizado é o tecnécio $99 \mathrm{~m}$ Tc. O exame avalia, em tempos pré-determinados, a presença do refluxo e seus graus - leve, moderado, grave - através da visualização do radioisótopo em esôfago distal, médio ou proximal, respectivamente. Além disso, é possível também verificar por quanto tempo esse fenômeno ocorre (o que pode sugerir um grau mais ou menos intenso de refluxo). O método permite também verificar se há aspiração pulmonar durante o refluxo, fato que pode ser fator desencadeante para doenças respiratórias como a asma. Em 14,8\% o exame não detectou refluxo gastroesofágico, sendo positivo em intensidade variáveis em todos os demais (85,2\%).

\section{CONCLUSÃO}

O exame subsidiário que apresentou elevada sensibilidade para o diagnóstico da esofagite de refluxo foi a endoscopia digestiva alta; o estudo cintilográfico foi o e que mais demonstrou refluxo gastroesofágico; o exame radiológico contrastado foi o de melhor eficácia em demonstrar o refluxo.

\section{REFERÊNCIAS}

1. Andreollo NA, Miquelino M, Lopes LR, Trevisan MAS, Brandalise NA, Leonardi LS. Incidência e epidemiologia do esôfago de Barrett no GASTROCENTRO-UNICAMP. Arq Bras Gastroent 1997; 34(1): 22-26.
2. Andreollo NA. Esofagite de refluxo. Quando e como operar? Arq Bras Cir Dig 1996: 11(1):28-29.

3. Andreollo NA, Thompson D, Kendall GPN, Mcintyre A, Earlam R. Motor responses of the upper esophageal sphincter and body to intraluminal acid. Brazilian J Med Biol Res 1989; 22:51-60.

4. Bais JE, Samsom M, Boudesteijn EAJ, Rijk PP, Akkermans LMA, Gooszen HG: Impact of delayed gastric emptying on the outcome of antireflux surgery. Ann f Surg 2001; 234:139-146.

5. Brito-Lugo P. Methodology in the study of gastroesophageal reflux disease and its complications. Rev Gastroenterol Mex. 2007;72 Suppl 2:9-12.

6. Cecconello I. Construção das Válvulas Anti-refluxo Gastroesofágico. In Aspectos Técnicos na Cirurgia do Aparelho Digestivo. Editado por Robe Livraria e Editora, São Paulo. pp5162, 1991.

7. Chen LQ Hu CH, Gaboury L, Pera M, Ferraro P, Duranceau AC. Proliferative activity in Barrett's esophagus before and after antireflux surgery. Ann Surg 2001; 234:172-180.

8. Domestic / International Gastroenterology Surveillance Study (DIGEST). Scand. J. Gastroenterol. 34:29-37, 1999.

9. Donahue PE. Basic considerations in gastroesophageal reflux disease. Surg Clin N Am 1997; 77:1017-1040.

10. horgan S, Pellegrini CA. Surgical treatment of gastroesophageal reflux disease. Surg Clin N Am 1997; 77:1063-1081.

11. Locke GR, Talley NJ, Fett SL, Zinsmeiter AR, Melton LJ: Prevalence and clinical spectrum of gastroesophageal reflux: a populationbased study in Olmsted county, Minnesota. Gastroenterology 1997; 112:1148-56.

12. Mittal RK. The crural diaphragm, an external lower esophageal sphincter: a definitive study. Gastroenterology 1993; 105: 1556-6.

13. Oliveira CF, Júnior GFL, Filho JSA. Contribuição da endoscopia ao Estudo da Doença do Refluxo Gastroesofágico. In Endoscopia Digestiva. Editado por Medsi Editora Médica e Científica S.A., Rio de Janeiro. pp 17-42, 1994

14. Ye P, Li ZS, Xu GM, Zou DW, Xu XR, Lu RH. Esophageal motility in patients with sliding hiatal hernia with reflux esophagitis. Chin Med J (Engl). 2008;20;121(10):898-903. 